\title{
Conjugacy in lattice-ordered groups and right ordered groups.
}

\author{
V. V. Bludov and A. M. W. Glass \\ November 5, 2007
}

\begin{abstract}
We prove that if $G_{j}$ are right orderable groups with non-trivial cyclic subgroups $C_{j}(j=1,2)$, then the (group) free product of $G_{1}$ and $G_{2}$ with $C_{1}$ and $C_{2}$ amalgamated is right orderable. We will deduce this from our main theorem that every lattice-ordered group can be embedded in one in which there are precisely four conjugacy classes. As a consequence of our method, we also show that every right ordered group can be embedded in a right ordered group in which any two non-identity elements are conjugate.
\end{abstract}

AMS Classification: 06F15, 20F60, 20B27, 20F10.

Keywords: amalgamation, free product with amalgamated subgroup, latticeordered group, right orderable group, permutation group, representation. 


\section{Background}

A group $G$ is said to be right orderable if there is a total order $\leq$ on $G$ such that $f h \leq g h$ whenever $f \leq g(f, g, h \in G)$.

A lattice-ordered group $G$ is a lattice and a group such that $h(f \vee g) k=$ $h f k \vee h g k$ and $h(f \wedge g) k=h f k \wedge h g k(f, g, h, k \in G)$. We denote by $G_{+}$the set $\{g \in G: g>1\}$ where $g \geq f$ is a shorthand for $g \vee f=g$. $G_{+}$is called the set of strictly positive elements of $G$. We write $g \| f$ if $(g \nsupseteq f$ and $g \not \leq f)$ and say that $g$ and $f$ are incomparable. If there are no elements incomparable to the identity, then the order is total and we say that the group is ordered. A group which can be made into an ordered group is called orderable.

Throughout, embeddings will be in context. For totally ordered sets they will preserve the order, for right orderable groups they will preserve the group operation, for right ordered groups they will preserve the group and order structure, and for lattice-ordered groups they will preserve the group and lattice operations.

If $(\Omega, \leq)$ is a totally ordered set, then $A(\Omega):=\operatorname{Aut}(\Omega, \leq)$ is a latticeordered group under composition and the pointwise lattice ordering $(\alpha(f \vee$ $g)=\max \{\alpha f, \alpha g\}$ and $\alpha(f \wedge g)=\min \{\alpha f, \alpha g\})$. Indeed, W. C. Holland [11] has proved that every lattice-ordered group can be embedded in $A(\Omega)$ for some totally ordered set $(\Omega, \leq)$, the embedding being as a lattice and a group. If $g \in A(\Omega)$, we let $\operatorname{supp}(g)=\{\alpha \in \Omega: \alpha g \neq \alpha\}$, the support of $g$. If $\alpha \in \operatorname{supp}(g)$, then the supporting interval of $g$ containing $\alpha$ is the set $\left\{\beta \in \Omega:(\exists m, n \in \mathbb{Z})\left(\alpha g^{n}<\beta<\alpha g^{m}\right)\right\}$. If $\alpha g>\alpha$, we say the interval is positive; and if $\alpha g<\alpha$, we say the interval is negative. A convex subset $\Delta$ of $\Omega$ that is maximal with respect to $\delta g=\delta(\delta \in \Delta)$ will be called a fixed-point interval of $g$.

Note that if $\Delta$ is a supporting interval of $g$, then there is a countable subset $\Delta_{0}=\left\{\alpha g^{n}: n \in \mathbb{Z}\right\}$ of $\Delta$ such that $\sup \left(\Delta_{0}\right)=\sup (\Delta)$ and $\inf \left(\Delta_{0}\right)=\inf (\Delta)$, the suprema and infima being in the Dedekind completion of $\Omega$.

Note that $A(\Omega)$ is right orderable: well order $(\Omega, \leq)$, say by $\prec$; declare $f \prec g$ in $G$ iff $\beta f<\beta g$, where $\beta$ is the least element (under $\prec$ ) of $\operatorname{supp}\left(g f^{-1}\right.$ ). This is a right ordering on $A(\Omega)$ that extends the lattice ordering on $A(\Omega)$; i.e., if $f<g$ in the lattice ordering, then $f \prec g$ in this right ordering (but not necessarily conversely). By Holland's Theorem, every lattice-ordered group is right orderable. Using the right regular representation, if $\leq$ is a right order on a group $G$, then $G$ can be embedded (as a group) in $\operatorname{Aut}(G, \leq)$. Hence every right orderable group can be embedded in a right orderable group that is lattice-orderable. We will exploit this duality. 
In [13], K. R. Pierce proved that the amalgamation property fails for lattice-ordered groups and V. V. Bludov [4] proved that the free product of right orderable groups with amalgamated subgroup need not be right orderable. In contrast, in the same paper (op. cit.), Pierce showed that one can amalgamate (in the class of lattice-ordered groups) a sublattice subgroup if it is an archimedean ordered group, and G. M. Bergman [2] gave conditions when a free product of right orderable groups with amalgamated subgroup is right orderable. Pierce's proof for the amalgamation of archimedean ordered sublattice subgroups relied on proving that every lattice-ordered group can be embedded in one in which any two strictly positive elements are conjugate. Since strictly negative elements are inverses of strictly positive ones and $g^{f}:=f^{-1} g f>1$ iff $g>1$, we have the number of conjugacy classes of a non-totally-ordered lattice-ordered group is at least four; it is at least three more than the number of conjugacy classes of elements incomparable to the identity element. Our main result is a strengthening of Pierce's result which answers Question 4 of the list of Unsolved Problems in [9], Chapter 11.

Theorem A Every lattice-ordered group can be embedded in one in which there are precisely four conjugacy classes.

We will deduce

Theorem B If $G_{j}$ are right orderable groups with non-trivial cyclic subgroups $C_{j}(j=1,2)$, then the (group) free product of $G_{1}$ and $G_{2}$ with $C_{1}$ and $C_{2}$ amalgamated is right orderable.

and

Theorem C Every right ordered group can be embedded in a right ordered group in which any two non-identity elements are conjugate.

Theorem C answers Question 5 of the list of Unsolved Problems in [9], Chapter 11.

\section{Proof of Theorem A}

The key to the modification of Pierce's proof is to represent $G$ as a group of order-preserving permutations so that for each $g \in G$ with $g \| 1$, between any two positive (negative) supporting intervals of $g$ there is both a positive and a negative supporting interval of $g$, and a (possibly singleton) fixed-point 
interval of $g$; moreover, such supporting intervals of $g$ occur above and below any positive or negative supporting interval of $g$ so that between them, the positive supporting intervals, negative supporting intervals and fixed point intervals of $g$ form an $\alpha$-set of degree 3 ; i.e., a saturated totally ordered 3coloured set (see [7] and below). We achieve this by modifying Pierce's proof. We will easily deduce that in some lattice-ordered group $G^{\dagger}$ containing $G$, there are precisely 4 conjugacy classes (in $G^{\dagger}$ ) of elements of $G$, and thence obtain Theorem A.

For ease of presentation, the proof that we give relies on the Generalised Continuum Hypothesis, though this dependence can be removed — see [14] and [15].

Recall that a totally ordered set $(\Lambda, \leq)$ of cardinality $\aleph_{\alpha}$ is called an $\alpha$-set if for all $\Delta_{1}, \Delta_{2} \subseteq \Lambda$ with $\Delta_{1}<\Delta_{2}$ and $\left|\Delta_{1} \cup \Delta_{2}\right|<\aleph_{\alpha}$, there is $\lambda \in \Lambda$ with $\Delta_{1}<\lambda<\Delta_{2}$. The existence of such sets for arbitrarily large $\alpha$ is guaranteed by the Generalised Continuum Hypothesis (see [7], Proposition 5.1.5).

Let $G$ be a lattice-ordered group. By Holland's Theorem, we may assume that $G$ is a sublattice subgroup of $A(\Omega)$ for some totally ordered set $(\Omega, \leq)$. We can embed $A(\Omega)$ in the group of order-preserving permutations of an $\alpha$ set, where $\aleph_{\alpha} \geq \max \left\{|G|,|\Omega|, \aleph_{1}\right\}$ - see [7], Propositions 5.1.8 and 5.1.9. We may therefore assume that $(\Omega, \leq)$ is an $\alpha$-set and $G$ is a sublattice subgroup of $A(\Omega)$. Let $(T, \leq)$ be another $\alpha$-set. Let $\tau_{1}^{\prime}<\tau_{1}<\tau_{2}<\tau_{2}^{\prime}$ in $\bar{T}$, the Dedekind completion of $T$, with $\tau_{1}, \tau_{1}^{\prime}$ the suprema of sequences of points in $T$ of order type strictly less than $\aleph_{\alpha}$ (and so the infima of sequences of points of $T$ of reverse order type $\aleph_{\alpha}$ and not less), and $\tau_{2}, \tau_{2}^{\prime}$ the infima of sequences of points in $T$ of reverse order type strictly less than $\aleph_{\alpha}$ (and so the suprema of sequences of points of $T$ of order type $\aleph_{\alpha}$ and not less). Then the intervals $\left(\tau_{1}, \tau_{2}\right)$ and $\left(\tau_{1}^{\prime}, \tau_{2}^{\prime}\right)$ in $T$ are $\alpha$-sets by definition. We identify $\Omega$ with the interval $\left(\tau_{1}, \tau_{2}\right)$ and so obtain that every element of $G$ has support (in $T$ ) bounded below by $\tau_{1}$ and above by $\tau_{2}$.

We recall the \# and b constructions from [8], pp. 193-201.

Let $(\Lambda, \leq)$ be a totally ordered set and $(\bar{\Lambda}, \leq)$ be the Dedekind completion of $(\Lambda, \leq)$ with the natural extended order. Let $\Lambda^{\#}$ be the union of $\Lambda$ and all cuts $(A, B) \subseteq \bar{\Lambda}$ where either there is a sequence in $A$ of cardinality strictly less than $\aleph_{\alpha}$ whose supremum is $\sup (A)=\inf (B)$ or there is a sequence in $B$ of cardinality less than $\aleph_{\alpha}$ whose infimum is $\sup (A)=\inf (B)$. Then $\Lambda^{\#}$ inherits the total order from $\bar{\Lambda}$. We regard $(\Lambda, \leq)$ as a subset of $\left(\Lambda^{\#}, \leq\right)$ in the natural way and embed $A(\Lambda)$ in $A\left(\Lambda^{\#}\right)$ by (uniquely) extending each orderpreserving permutation of $(\Lambda, \leq)$ to one of $\left(\Lambda^{\#}, \leq\right)$. If a lattice-ordered group 
$H$ is a sublattice subgroup of $A(\Lambda)$, then we denote by $H^{\#}$ its extension to a sublattice subgroup of $A\left(\Lambda^{\#}\right)$. So $H \cong H^{\#}$.

The $b$ construction is more complicated and we will need to modify it.

Let $(\Lambda, \leq)$ be a totally ordered set and $\sigma_{1}<\sigma_{2}$ in $\Lambda$. Let $H$ be a sublattice subgroup of $A(\Lambda)$ with $\operatorname{supp}(h) \subseteq\left(\sigma_{1}, \sigma_{2}\right)$ for all $h \in H$. Let $\mathcal{K}$ be the set of $H$-orbits of $\Lambda$. For each $K \in \mathcal{K}$, choose $0_{K} \in K$. For each $\beta \in K$, pick $c_{\beta} \in H$ so that $0_{K} c_{\beta}=\beta$. We require that $c_{0_{K}}$ be the identity. For $\lambda_{1}, \lambda_{2} \in K$, define $c_{\lambda_{1}, \lambda_{2}}:=c_{\lambda_{1}}^{-1} c_{\lambda_{2}} ;$ so $\lambda_{1} c_{\lambda_{1}, \lambda_{2}}=\lambda_{2}$. Let

$$
\begin{gathered}
L_{K}:=\left\{h \in H:\left(\exists \lambda<0_{K}\right)\left(\operatorname{supp}(h) \cap\left[\lambda, 0_{K}\right]=\emptyset\right)\right\} \text { and } \\
R_{K}:=\left\{h \in H:\left(\exists \lambda>0_{K}\right)\left(\operatorname{supp}(h) \cap\left[0_{K}, \lambda\right]=\emptyset\right)\right\} .
\end{gathered}
$$

Then $L_{K}$ and $R_{K}$ are $\ell$-ideals (convex normal sublattice subgroups) of the stabiliser subgroup $H_{0_{K}}:=\left\{h \in H: 0_{K} h=0_{K}\right\}$. Clearly, $H_{0_{K}} / L_{K}$ and $H_{0_{K}} / R_{K}$ are lattice-ordered groups under the inherited order. By Holland's Theorem and [8] Corollary 2.L, for each $K \in \mathcal{K}$ there are totally ordered sets $\left(\Gamma_{j}(K), \leq\right)$ with $\left|\Gamma_{j}(K)\right| \leq|H|(j=1,2)$ and $H_{0_{K}} / L_{K}$ a sublattice subgroup of $B\left(\Gamma_{1}(K)\right)$ and $H_{0_{K}} / R_{K}$ a sublattice subgroup of $B\left(\Gamma_{2}(K)\right)$, where

$$
B(\Omega)=\left\{g \in A(\Omega):\left(\exists \beta_{1}<\beta_{2} \text { in } \Omega\right) \operatorname{supp}(g) \subseteq\left(\beta_{1}, \beta_{2}\right)\right\} .
$$

If $\Delta_{1}, \Delta_{2}$ are disjoint totally ordered sets, we write $\Delta_{1} \overleftarrow{\cup} \Delta_{2}$ for $\Delta_{1} \cup \Delta_{2}$ totally ordered by $\Delta_{1}<\Delta_{2}$.

Let $\{\xi(K): K \in \mathcal{K}\}$ be a set of new symbols. Pierce let

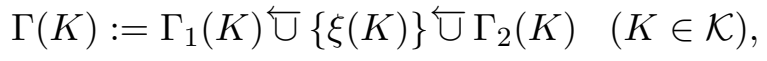

and we do the same - but only if $K$ (necessarily the singleton $\left\{0_{K}\right\}$ ) satisfies $K \cap\left(\sigma_{1}, \sigma_{2}\right)=\emptyset$. However, we modify his construction if $K \in \mathcal{K}$ with $K \subseteq$ $\left(\sigma_{1}, \sigma_{2}\right)$ by instead letting $\{\Lambda(K): K \in \mathcal{K}\}$ be a disjoint family of copies of $\Lambda$ (under the identification $\lambda \mapsto \lambda(K)$ ) and defining

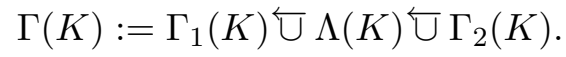

$$
\begin{aligned}
& \text { Let } \Lambda^{b}:=\{(\gamma, \lambda): \lambda \in K \in \mathcal{K}, \gamma \in \Gamma(K)\} \text {. }
\end{aligned}
$$

Define a total order on $\Lambda^{\text {b }}$ by:

$$
\left(\gamma_{1}, \lambda_{1}\right)<\left(\gamma_{2}, \lambda_{2}\right) \text { if } \lambda_{1}<\lambda_{2} \text { in } \Lambda \text { or }\left(\lambda_{1}=\lambda_{2} \in K \in \mathcal{K} \text { and } \gamma_{1}<\gamma_{2} \text { in } \Gamma(K)\right) \text {. }
$$

We embed $\Lambda$ in $\Lambda^{b}$ by $\varphi^{b}$ :

$$
\lambda \varphi^{b}= \begin{cases}(\lambda(K), \lambda) & \text { if } \sigma_{1}<\lambda<\sigma_{2} \text { and } \lambda \in K \in \mathcal{K} \\ (\xi(K), \lambda) & \text { if } \lambda \notin\left(\sigma_{1}, \sigma_{2}\right) \text { and } \lambda=0_{K}\end{cases}
$$


and embed $H$ in $A\left(\Lambda^{b}\right)$ by $\psi^{b}$ where (for $h \in H ; \lambda \in K \in \mathcal{K}, \gamma(K) \in \Gamma(K)$ ) we define $(\gamma(K), \lambda h)\left(h \psi^{b}\right)$ by

$$
\begin{cases}\left(\gamma(K) c_{\gamma(K)} h c_{\gamma(K) h}^{-1} L_{0_{K}}, \lambda h\right) & \text { if } \sigma_{1}<\lambda<\sigma_{2} \text { and } \gamma(K) \in \Gamma_{1}(K) \\ ((\gamma h)(K), \lambda h) & \text { if } \sigma_{1}<\lambda<\sigma_{2} \text { and } \gamma(K) \in \Lambda(K) \\ \left(\gamma(K) c_{\gamma(K)} h c_{\gamma(K) h}^{-1} R_{0_{K}}, \lambda h\right) & \text { if } \sigma_{1}<\lambda<\sigma_{2} \text { and } \gamma(K) \in \Gamma_{2}(K) \\ (\gamma(K), \lambda) & \text { if } \lambda \notin\left(\sigma_{1}, \sigma_{2}\right) .\end{cases}
$$

We let $H^{b}:=H \psi^{b}$.

By construction, $\left(\lambda \varphi^{b}\right)\left(h \psi^{b}\right)=(\lambda h) \varphi^{b}$; so $\left(\lambda \varphi^{b}\right)\left(h \psi^{b}\right)=\lambda \varphi^{b}$ iff $\lambda h=\lambda$ $(h \in H ; \lambda \in \Lambda)$.

We now alternate these two constructions to form $\left(G^{\dagger}, T^{\dagger}\right)$ from $(G, \Omega)$ by transfinite induction.

Let $\left(G_{0}, \Omega_{0}\right):=(G, T)$ as above with $\operatorname{supp}(g) \subseteq\left(\tau_{1}, \tau_{2}\right)$ for all $g \in G$.

Let $\left\{\tau_{1, \mu}: \mu<\aleph_{\alpha}\right\}$ be an ordinally indexed strictly decreasing sequence with $\inf \left\{\tau_{1, \mu}: \mu<\aleph_{\alpha}\right\}=\tau_{1}^{\prime}, \tau_{1,0}=\tau_{1}$ and for any limit ordinal $\mu<\aleph_{\alpha}$, let $\tau_{1, \mu}=\inf \left\{\tau_{1, \nu}: \nu<\mu\right\}$. Dually, let $\left\{\tau_{2, \mu}: \mu<\aleph_{\alpha}\right\}$ be an ordinally indexed strictly increasing sequence with $\sup \left\{\tau_{2, \mu}: \mu<\aleph_{\alpha}\right\}=\tau_{2}^{\prime}, \tau_{2,0}=\tau_{2}$ and for any limit ordinal $\mu<\aleph_{\alpha}$, let $\tau_{2, \mu}=\sup \left\{\tau_{2, \nu}: \nu<\mu\right\}$.

Every successor ordinal can be written in the form $\nu+n$, where $\nu$ is a limit ordinal or 0 and $n \in \mathbb{Z}_{+}$.

If $n$ is odd, say $n=2 m+1$ so $\mu=\nu+2 m+1$, let $\left(G_{\mu}, T_{\mu}\right):=\left(G_{\nu+2 m}^{\#}, T_{\nu+2 m}^{\#}\right)$, $\varphi_{\mu}$ be the identity on $T_{\nu+2 m}$ and $\psi_{\mu}$ be $\psi^{\#}$ applied to $G_{\nu+2 m}$.

If $n$ is even, say $n=2 m$ so $\mu=\nu+2 m$, we apply the $b$ construction to $\left(G_{\nu+2 m-1}, T_{\nu+2 m-1}\right)$ with $\sigma_{j}:=\tau_{j, \nu+2 m}(j=1,2)$, and let $\left(G_{\mu}, T_{\mu}\right):=$ $\left(G_{\nu+2 m-1}^{b}, T_{\nu+2 m-1}^{b}\right), \varphi_{\mu}$ be $\varphi^{b}$ applied to $T_{\nu+2 m-1}$ and $\psi_{\mu}$ be $\psi^{b}$ applied to $G_{\nu+2 m-1}$.

If $\mu$ is a limit ordinal, let $\left(G_{\mu}, T_{\mu}\right)$ be the direct limit of $\left\{\left(G_{\mu^{\prime}}, T_{\mu^{\prime}}\right): \mu^{\prime}<\right.$ $\mu\}$. Let $\varphi_{\mu}$ be the direct limit of $\left\{\varphi_{\mu^{\prime}}: \mu^{\prime}<\mu\right\}$ and $\psi_{\mu}$ be the direct limit of $\left\{\psi_{\mu^{\prime}}: \mu^{\prime}<\mu\right\}$.

Let $\left(T^{\dagger}, \leq\right)$ be the direct limit of $\left\{\left(T_{\mu}, \leq\right): \mu<\aleph_{\alpha}\right\}$ with the above order-preserving injections. Note that $T^{\dagger}$ is an $\alpha$-set as it has the desired properties by construction. We view $G^{*}$, the direct limit of $\left\{G \psi_{\mu}: \mu<\aleph_{\alpha}\right\}$, as a sublattice subgroup of $A\left(T^{\dagger}\right)$ in the natural way. By construction, every element of $G^{*}$ has support contained in the interval $\left(\tau_{1}^{\prime}, \tau_{2}^{\prime}\right)$ of $T^{\dagger}$.

By construction, if $I$ is a non-empty collection of supporting intervals of $g \psi_{\mu}$ with $\mu=\nu+2 m$ ( $\nu$ a limit ordinal or $\left.0 ; m \in \mathbb{Z}_{+} \cup\{0\}\right)$ and $\inf (\bigcup I)$ exists 
in $T_{\mu+1}$ (say, $\sigma$ ), then there are distinct intervals of support and fixed point intervals of $g \psi_{\mu+2}$ in $(\sigma, \inf (\bigcup I))$ (and dually if $\sup (\bigcup I)$ exists in $\left.T_{\mu+1}\right)$.

By construction, if $\mu<\aleph_{\alpha}, g \in G \backslash\{1\}$ and $\Delta_{1}<\Delta_{2}$ in $T_{\mu}$ are supporting intervals of $g \psi_{\mu}$, then since $\Delta_{1}$ has a countable sequence with supremum (in the Dedekind completion of $\left.T_{\mu}\right) \sup \left(\Delta_{1}\right)$ and dually for $\Delta_{2}$, then there is $\lambda \in T_{\mu^{\prime}}$ with $\Delta_{1}<\lambda<\Delta_{2}$, where $\mu^{\prime}$ is the least odd ordinal greater than $\mu$. Let $K$ be the $G \psi_{\mu^{\prime}}$-orbit to which $\lambda$ belongs, and observe that $\tau_{1, \mu^{\prime}+1}<$ $\lambda<\tau_{2, \mu^{\prime}+1}$. So the action of $g \psi_{\mu^{\prime}+1}$ on $T_{\mu^{\prime}}(K)$ is isomorphic to the action of $g \psi_{\mu^{\prime}}$ on $T_{\mu^{\prime}}$. Hence the interval $\left(\sup \left(\Delta_{1}\right), \inf \left(\Delta_{2}\right)\right)$ in $T_{\mu^{\prime}+1}$ includes supporting intervals and fixed-point intervals of $g \psi_{\mu^{\prime}+1}$, where the supremum and infimum of $\Delta_{1}, \Delta_{2}$ are taken in the Dedekind completion of $T_{\mu^{\prime}}$; if $g \| 1$, there are positive and negative supporting intervals contained in this interval $\left(\sup \left(\Delta_{1}\right), \inf \left(\Delta_{2}\right)\right)$ of $T_{\mu^{\prime}+1}$. Also note that there is an interval of support of $g \psi_{\mu^{\prime}+1}$ above $\tau_{2, \mu^{\prime}}$ (and so above $\Delta_{2}$ ) and an interval of support of $g \psi_{\mu^{\prime}+1}$ below $\tau_{1, \mu^{\prime}}$ (and so below $\Delta_{1}$ ); if additionally $g \| 1$ and the hypotheses pertain, then there are positive and negative supporting intervals $\Delta_{+}, \Delta_{-}$of $g \psi_{\mu^{\prime}+1}$ above $\Delta_{2}$ (and mutatis mutandis below $\Delta_{1}$ ).

However, in contrast to Pierce's construction, if $\mu$ is a limit ordinal, then all fixed point intervals of $g \psi_{\mu}(\neq 1)$ contained in $\left(\tau_{1, \mu}, \tau_{2, \mu}\right)$ are singletons. For if $\tau_{1, \mu} \leq \lambda_{1}<\lambda_{2} \leq \tau_{2, \mu}$ with $\lambda_{j} g \psi_{\mu}=\lambda_{j}(j=1,2)$, then $\lambda_{j} \in T_{\nu}$ for some odd ordinal $\nu<\mu$ and, as noted above, $\lambda_{j} \varphi_{\nu+1}$ is fixed by $g \psi_{\nu+1}(j=1,2)$. Let $\lambda \in T_{\nu+1}$ with $\lambda_{1}<\lambda<\lambda_{2}$ and $K$ be the $G_{\nu+1} \psi_{\nu+1}$-orbit to which $\lambda$ belongs. Then $\lambda_{1} \varphi^{b}<\left(T_{\nu+1}(K), \lambda\right)<\lambda_{2} \varphi^{b}$ and $g \psi_{\nu+2}$ is not the identity on $\left(T_{\nu+1}(K), \lambda\right)$. Hence $g \psi_{\mu}$ is not the identity on $\left(\lambda_{1}, \lambda_{2}\right) \subseteq T_{\mu}$.

Recall that a 3 -coloured $\alpha$-set is an $\alpha$-set $\Omega$, each of whose elements are coloured exactly one of either Red, Amber or Green such that for any $B_{1}, B_{2} \subseteq$ $\Omega$ with $B_{1}<B_{2}$ and $\left|B_{1} \cup B_{2}\right|<\aleph_{\alpha}$, there are points $\gamma_{R}, \gamma_{A}$ and $\gamma_{G}$ coloured Red, Amber and Green, respectively, such that $B_{1}<\gamma_{j}<B_{2}$ for $j \in$ $\{R, A, G\}$. This definition allows $B_{1}$ or $B_{2}$ to be empty. Let $g^{*}:=g \psi_{\aleph_{\alpha}}$ be a non-identity element of $G^{*}$. The above computations show that if we colour the positive intervals of $g^{*}$ Green, the negative intervals of $g^{*}$ Amber and the fixed points of $g^{*}$ Red, then

(i) if $g>1$, the set of intervals of $g^{*}$ coloured Red and Green forms a 2-coloured $\alpha$-set;

(ii) if $g<1$, the set of intervals of $g^{*}$ coloured Red and Amber forms a 2 -coloured $\alpha$-set; and

(iii) if $g \| 1$, the set of intervals of $g^{*}$ coloured Red, Amber and Green forms a 3-coloured $\alpha$-set.

Since 2 coloured $\alpha$-sets and 3 coloured $\alpha$-sets form saturated models, each 
is homogeneous ([7] Proposition 5.1.9). Hence, if $g, h \in G_{+}$, there is $f \in A\left(T^{\dagger}\right)$ with $f^{-1} g f=h$; if $g, h<1$, there is $f \in A\left(T^{\dagger}\right)$ with $f^{-1} g f=h$; and if $g, h \| 1$, there is $f \in A\left(T^{\dagger}\right)$ with $f^{-1} g f=h$.

Enumerate all pairs $P$ of elements of $G^{*}$ that are both strictly positive; do the same for all pairs $N$ of $G^{*}$ that are both strictly negative, and also for all pairs $I$ of $G^{*}$ that are both incomparable to the identity. The number of such pairs is $|G|$. For each such pair, $(g, h)$, let $f:=f(g, h) \in A\left(T^{\dagger}\right)$ be such that $f^{-1} g f=h$. Let $G^{\dagger}$ be the sublattice subgroup of $A\left(T^{\dagger}\right)$ generated by $G^{*}$ and the set of all $f(g, h)$ for $(g, h) \in P \cup N \cup I$. Then $\left|G^{\dagger}\right| \leq \aleph_{\alpha}$ and $G^{*} \cong G$ has precisely 4 conjugacy classes in $G^{\dagger}$.

[Aside: For each pair $(g, h) \in P \cup N \cup I$, we can choose $f \in A\left(T^{\dagger}\right)$ with $f^{-1} g f=h$ so that (if $\left.g \not \leq 1\right) f$ moves uncountably many positive intervals of $g$ up and also uncountably many such intervals down; and further (if $g \nsupseteq 1$ ) $f$ moves uncountably many negative intervals of $g$ up and also uncountably many such intervals down. This follows immediately by the high homogeneity of saturated models (op. cit.).]

Let $G(0):=G$; and for $m \in \mathbb{Z}_{+} \cup\{0\}$, let $G(m+1):=G(m)^{\dagger}$. Let $L:=\bigcup\left\{G(m): m \in \mathbb{Z}_{+} \cup\{0\}\right\}$. By construction, $L$ has precisely 4 conjugacy classes and contains $G$ as a sublattice subgroup.

This completes the proof of Theorem A. //

Corollary 2.1 Every lattice-ordered group $G$ can be embedded in a latticeordered group $L$ which has the property that for all $h_{0} \in L \backslash\{1\}$, each element of $L$ is the product of a conjugate of $h_{0}$ and a conjugate of $h_{0}^{-1}$.

The following proof is due to George M. Bergman and simplifies our original. We are most grateful to him for allowing us to use it.

Proof: Let $L$ be the lattice-ordered group constructed from $G$ in Theorem A, and $h_{0} \in L \backslash\{1\}$. Let $C$ be the conjugacy class to which $h_{0}$ belongs. If $g \in C \cup C^{-1}$, the result is immediate since $g=g^{2} g^{-1}=g^{-1} g^{2}$. Since every $g \in L$ can be written as $(g \vee 1)\left(g^{-1} \vee 1\right)^{-1}=\left(g^{-1} \vee 1\right)^{-1}(g \vee 1)$ ([9], Lemma 2.3.8), every element of $L$ can be written in the desired form if $h_{0}>1$. A similar argument applies if $h_{0}<1$. We now consider the remaining case that $h_{0} \| 1$ and $g \notin C$. Let $f \in L$ be such that $f^{-1}\left(h_{0} \vee 1\right) f=\left(h_{0} \vee 1\right)^{2}$. Then $h_{1}:=f\left(h_{0} \vee 1\right) f^{-1}$ is such that $1<h_{1}<h_{1}^{2}=h_{0} \vee 1$. Now $h_{1}^{-1} h_{0} \in C$ and $h_{1}=h_{0} \cdot\left(h_{1}^{-1} h_{0}\right)^{-1}$, so there is a strictly positive element (namely, $h_{1}$ ) that is a product of a conjugate of $h_{0}$ and a conjugate of $h_{0}^{-1}$. Hence every strictly positive element of $L$ can be written in the desired form. Since $C=C^{-1}$ in 
this case, every strictly negative element of $L$ can be written in the desired form. The corollary follows. //

Corollary 2.2 Let $G$ be a finitely generated lattice-ordered group. Then $G$ has soluble word problem if and only if it can be embedded in a simple latticeordered group that can be embedded in a finitely presented lattice-ordered group.

Proof: In [10] A. M. W. Glass proved that if $G$ is any finitely generated lattice-ordered group, then $G$ has soluble word problem if and only if it can be embedded in an $\ell$-simple lattice-ordered group $S$ that can be embedded in a finitely presented lattice-ordered group. The lattice-ordered group $S$ had the property that any two strictly positive elements of $S$ were conjugate. However, if $G$ has soluble word problem and we use $G(0)$ of the proof of Corollary 2.1, then $G(0)$ is finitely generated and has soluble word problem. Instead of using Pierce's result, we apply the proof of Theorem A (together with the aside) to $G(0)$ in the construction in [10] (substituting $P \cup N \cup I$ in place of $P$ ) and obtain that $S$ has exactly 4 conjugacy classes; it is simple as a group by the proof of Corollary 2.1. //

\section{Deduction of Theorems B and C}

Proof of Theorem B: Let $j=1,2$ and $G_{j}$ be a right orderable group with non-trivial cyclic subgroup $C_{j}$. Say, $C_{j}=\left\langle f_{j}\right\rangle$ with $f_{j} \neq 1$. Let $\leq_{j}$ be a right order on $G_{j}$ and $\leq_{j}^{\prime}$ be the reverse order. Let $\left(\Omega_{j}, \leq\right):=\left(G_{j}, \leq_{j}\right)$ and $\left(\Omega_{j}^{\prime}, \leq_{j}^{\prime}\right)$ be a disjoint copy of $\left(G_{j}, \leq_{j}^{\prime}\right)$. Then the disjoint union $T_{j}$ of $\left(\Omega_{j}, \leq\right)$

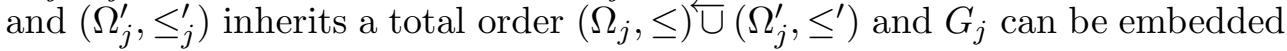
in the group of order-preserving permutations of $T_{j}$ by using the right regular representation on each (any point $\alpha \in \Omega_{j}$ is mapped by $g \in G_{j}$ to their product $\alpha g$ in $\Omega_{j}=G_{j}$; any point $\alpha^{\prime} \in \Omega_{j}^{\prime}$ is mapped by $g \in G_{j}$ to the image of their product $(\alpha g)^{\prime}$ in $\Omega_{j}^{\prime}$ (under the natural maps)). Note that every non-identity element of $G_{j}$ is mapped to an element of $A\left(T_{j}\right)$ that is incomparable to the identity element in the pointwise lattice ordering as $1 g<1$ iff $1^{\prime}<1^{\prime} g$. Let $T:=T_{1}^{\overleftarrow{U}} T_{2}$. Then $G_{1} \times G_{2}$ can be embedded as a group in the lattice-ordered group $A(T)$ in the natural way so that $\operatorname{supp}\left(g_{j}\right) \subseteq T_{j}(j=1,2)$. Then the images of $f_{1}$ and $f_{2}$ are incomparable to the identity. By Theorem A, there is an embedding of $A(T)$ into a lattice-ordered group $L$ in which the images of $f_{1}$ and $f_{2}$ are conjugate, say by $y \in L$. Let $\psi_{j}: G_{j} \rightarrow L$ be the embeddings $(j=1,2)$ and $\hat{y}: x \mapsto y^{-1} x y$ be the inner automorphism of $L(x \in L)$. Then 
the embeddings $\psi_{1} \hat{y}: G_{1} \rightarrow L$ and $\psi_{2}: G_{2} \rightarrow L$ amalgamate the images of $f_{1}$ and $f_{2}$ in $L$. As shown in Section 1 , every lattice-ordered group is right orderable. Hence so is $L$. That is, the right orderable groups $G_{1}$ and $G_{2}$ can be amalgamated over $C_{1}, C_{2}$ in the class of right orderable groups. It follows by Bergman's result ([2], Theorem 35) that the (group) free product of $G_{1}$ and $G_{2}$ with $C_{1}$ and $C_{2}$ amalgamated is also right orderable. //

The above proof can be adapted to show that any right orderable group can be embedded in a right orderable group in which any two non-identity elements are conjugate. Rather than give this modification, we prove a stronger version, Theorem C.

Proof of Theorem C: As in the penultimate paragraph of the proof of Theorem A, it is enough to prove that every right ordered group $(G, \leq)$ can be embedded in a right ordered group $(r(L), \leq)$ in which any two non-identity elements of $G$ are conjugate. Let $(G, \leq)$ be a right ordered group. Let $\left(G^{\prime}, \leq^{\prime}\right)$ be a copy of the same group but with the reverse right order. Let $(\Omega, \leq)$ be

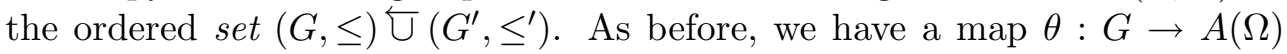
given by:

$$
\alpha(g \theta)=\alpha g \quad(\alpha \in G) \quad \text { and } \quad \alpha^{\prime}(g \theta)=(\alpha g)^{\prime} \quad\left(\alpha^{\prime} \in G^{\prime}\right) .
$$

Then $\theta$ is a group embedding of $G$ into $A(\Omega)$. If $a \in G_{+}$, then under the pointwise (lattice) ordering on $A(\Omega)$ we have that $a \theta \| 1$ since $1(a \theta)=a \geq 1$ and $1^{\prime}(a \theta)=a^{\prime} \leq^{\prime} 1^{\prime}$; and mutatis mutandis if $a^{-1} \in G_{+}$. Thus the image under $\theta$ of any non-identity element of $G$ is incomparable to the identity of the lattice-ordered group $A(\Omega)$. We now proceed as in the proof of Theorem $\mathrm{A}$ to get a lattice-ordered group $L$ (with $A(\Omega)$ isomorphic to a sublattice subgroup of $L$ which is a sublattice subgroup of $A(T))$ in which any pair of elements of $A(\Omega)$ that are incomparable to the identity are conjugate in $L$. Let $\psi$ denote this embedding. By construction, any two non-identity elements of $G$ are conjugate in $L$. In the construction, we obtained an order-preserving injection $\varphi:(\Omega, \leq) \rightarrow(T, \leq)$ with

$$
(\alpha \varphi)(g \theta \psi)=(\alpha(g \theta)) \varphi \quad(\alpha \in \Omega, g \in G) .
$$

We now change the orderings on $A(\Omega)$ and $A(T)$. Well order $\Omega$ so that the identity element of $G$ is the least element, and right order $A(\Omega)$ as explained in Section 1 . Then $\theta$ preserves the right order $(G, \leq)$ because the identity of $G$ is the least element of $\Omega$ in the well ordering. If we well order $T$ with least element $1 \varphi$, then $A(T)$ (and so $L$ ) becomes a right ordered group. We denote the group $L$ with this right ordering by $(r(L), \leq)$. Now $\theta \psi:(G, \leq) \rightarrow(r(L), \leq)$ is an 
embedding of $(G, \leq)$ in $(r(L), \leq)$ (as right ordered groups). This establishes Theorem C. //

Question 5 in [9], Chapter 11 only asked if every right ordered group can be embedded in one in which any two strictly positive elements are conjugate. So Theorem C does more.

Corollary 3.1 Every ordered group can be embedded in a right ordered group in which any two non-identity elements are conjugate.

This corollary may at first seem surprising since it is false if we replace the category of right ordered groups by either the category of ordered groups or the category of lattice-ordered groups $\left(g^{-1} f g>1\right.$ whenever $f>1$ if the order is two sided). Indeed, the corollary is false if we restrict to the class of ordered groups and merely require that any two strictly positive elements are conjugate. This is immediate because the larger group is necessarily divisible (for any $m \in \mathbb{Z}_{+}$and $g \in L$, there is $f_{m} \in L$ such that $f_{m}^{-1} g f_{m}=g^{m}$; so $f_{m} g f_{m}^{-1}$ is an $m^{t h}$ root of $g$ ) and V. V. Bludov has provided examples of centre-by-metabelian orderable groups which cannot be embedded in any divisible orderable group [3]. However, the corollary does give another proof of the known result ([9], Corollary 8.6.4) that every right ordered group can be embedded in a divisible right ordered group.

\section{Equations over groups}

Let $G$ be a group and $F=F\left(x_{1}, \ldots, n\right)$ be the free group of rank $n$. Let $H:=G * F$. Let $w=w\left(a_{1}, \ldots, a_{k}, x_{1}, \ldots, x_{n}\right) \in H$ with $a_{1}, \ldots, a_{k} \in G$ and $x_{1}, \ldots, x_{n} \in F$. We call $x_{1}, \ldots, x_{n}$ the variables in $w$ and $a_{1}, \ldots, a_{k}$ the constants in $w$. For example, $u(a, x):=a x^{-1} a x$ has one variable $x$ and one constant $(a \in G)$. Suppose that $w$ not conjugate to any element of $G$; i.e., $w \in H \backslash G^{H}$. We obtain an equation $w\left(a_{1}, \ldots, a_{k}, x_{1}, \ldots, x_{n}\right)=1$ over the group $G$ in variables $x_{1}, \ldots, x_{n}$ with constants $a_{1}, \ldots, a_{k}$ (in $G$ ). We say that the equation $w=1$ is soluble over $G$ if $G$ is embeddable in a group $\hat{G}$ containing elements $\hat{g}_{1}, \ldots, \hat{g}_{n}$ such that $w\left(a_{1}, \ldots, a_{k}, \hat{g}_{1}, \ldots, \hat{g}_{n}\right)=1$ in $\hat{G}$; and we say that the equation $w=1$ is soluble in $\hat{G}$ and that $\left(\hat{g}_{1}, \ldots, \hat{g}_{n}\right)$ is a solution.

A class of groups $\mathfrak{K}$ is closed under equation $w=1$ if for any group $G \in \mathfrak{K}$, there is a group $\hat{G} \in \mathfrak{K}$ containing $G$ in which the equation $w=1$ is soluble.

Since every right ordered group can be embedded in a divisible right ordered group (Theorem C or [9], Corollary 8.6.4), the classes of right ordered and right orderable groups are closed under equations $a x^{n}=1, n \in \mathbb{Z}_{+}$. Also, 
from Theorem $\mathrm{C}$ we deduce that the classes of right ordered and right orderable groups are closed under equation $a_{1} x^{-1} a_{2} x=1\left(a_{1}, a_{2} \neq 1\right)$. Of course, $a x^{-1} a x=1$ has no solution in any lattice-ordered group if $a>1$. However,

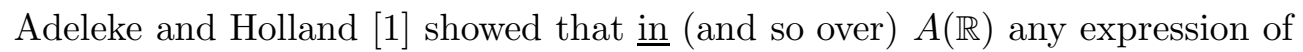
the form $w\left(x_{1}, \ldots, x_{n}\right)=a$ (with constant $\left.a \in A(\mathbb{R})\right)$ has a solution in $A(\mathbb{R})$. Note that if $G$ is any right ordered group and $a \in G_{+}$, then $a x^{-1} a x=1$ is soluble in a right ordered group containing $G$ by Corollary 3.1. We can say a little more:

Theorem D The classes of right ordered and right orderable groups are closed under any equation $w\left(a, x_{1}, \ldots, x_{n}\right)=1$ with a single constant $(a \in G)$.

Proof: We may assume that $w\left(a, x_{1}, \ldots, x_{n}\right)$ is not a proper power (if $w\left(a, x_{1}, \ldots, x_{n}\right)=v\left(a, x_{1}, \ldots, x_{n}\right)^{m}$, then the solutions to $w=1$ and $v=$ 1 are the same). In this case (see [12], Proposition 5.18), the one-relator group $L=\left\langle b, y_{1}, \ldots, y_{n} \mid w\left(b, y_{1}, \ldots, y_{n}\right)=1\right\rangle$ is torsion-free. By a result of S. D. Brodsky ([5], Corollary 2.3$), H$ is locally indicable and so right orderable [6]. Now let $(G, \leq)$ be any right ordered group. We can embed $G$ (extending the order) into the direct product $G \times L$ and (by Theorem C) then embed $G \times L$ into a right ordered group $\hat{G}$ in which the elements $a \in G$ and $b \in L$ are conjugate: $a=b^{h}$ for a suitable $h \in \hat{G}$. Since $w\left(b, y_{1}, \ldots, y_{n}\right)=1$, we have that $\left(y_{1}^{h}, \ldots, y_{n}^{h}\right)$ is a solution to the equation $w\left(a, x_{1}, \ldots, x_{n}\right)=1$ in $\hat{G}$. //

In the general case (when there are more constants), are there always solutions of equations over right ordered (right orderable) groups?

\section{References}

[1] S. A. Adeleke and W. C. Holland, Representations of order automorphisms by words, Forum Math. 6 (1994), 315-321.

[2] G. M. Bergman, Ordering coproducts of groups and semigroups, J. Algebra 133 (1990), 313-339.

[3] V. V. Bludov, On the completion of linearly ordered groups, Algebra i Logika 44 (2005), 664-681, 764; English translation: 370-380.

[4] V. V. Bludov, On the free product of right ordered groups with amalgamated subgroup, Problems of modern mathematics, Proceedings of scientific works, V. II, NII MIOO NGU, Novosibirsk, 1966, 30-35. 
[5] S. D. Brodsky, Equations over groups and groups with one defining relation, Siberian Math. J. XXV, No 2 (1984), 84-103 (in Russian); English translation: 235-251.

[6] R. G. Burns and V. W. Hale, A note of groups rings of certain torsion-free groups, Canad. Math. Bull., 1972, 15 (1972), 441-445.

[7] C. C. Chang and H. J. Keisler, Model Theory, North Holland Pub. Co., Amsterdam, 1973.

[8] A. M. W. Glass, Ordered Permutation Groups, London Math. Soc. Lecture Notes Series 55 (1981), Cambridge University Press, Cambridge.

[9] A. M. W. Glass, Partially Ordered Groups, Series in Algebra 7, World Scientific Pub. Co., Singapore, 1999.

[10] A. M. W. Glass, Finitely generated lattice-ordered groups with soluble word problem, J. Group Theory (to appear).

[11] W. C. Holland, The lattice-ordered group of automorphisms of an ordered set, Michigan Math. J. 10 (1963), 399-408.

[12] R. C. Lyndon and P. E. Schupp, Combinatorial Group Theory, Ergebnisse der Math. und ihrer Grenzgebiete 89, Springer-Verlag, Heidelberg, 1977.

[13] K. R. Pierce, Amalgamations of lattice-ordered groups, Trans. American Math. Soc. 172 (1972), 249-260.

[14] E. C. Weinberg, Automorphism groups of minimal $\eta_{\alpha}$-sets, in Ordered Groups: Proc. Boise Conference 1978 (ed. J. E. Smith, G. O. Kenney and R. N. Ball), Marcel Dekker, New York, 1980, 71-79; and

[15] E. C. Weinberg, Partitioned chains and amalgamations of lattice-ordered groups, (unpublished manuscript).

Authors' addresses:

V. V. Bludov:

Department of Mathematics, Physics, and Informatics, Irkutsk Teachers Training University,

Irkutsk 664011,

Russia

vasily-bludov@yandex.ru 
A. M. W. Glass:

Department of Pure Mathematics and Mathematical Statistics, Centre for Mathematical Sciences,

Wilberforce Rd.,

Cambridge CB3 0WB,

England

amwg@dpmms.cam.ac.uk 